\title{
I mplementing combined decision models in healthcare settings: the Simon and Pauker- Kassirer models
}

\author{
Ofir Ben-Assuli \\ Faculty of Business Administration, Ono Academic College, Kiryat Ono, Israel
}

Correspondence: Ofir Ben-Assuli. Vice president of medical informatics, The Center for Medical Decision Making, Ono Academic College. Address: Faculty of Business Administration, Ono Academic College, 104 Zahal st., Kiryat Ono, Israel. E-mail: ofir.benassuli@gmail.com

Received: July 28, 2013

DOI : $10.5430 /$ jha.v3n2p10
Accepted: October 14, 2013

URL: http://dx.doi.org/10.5430/jha.v3n2p10

\section{Abstract}

Background: Modeling medical decision-making has attracted considerable attention over the years, and has become the topic of many investigations. Researchers have attempted to model this critical and extremely complex process from several different angles to enable hospital clinicians to engage in decision-making using empirical tools.

Purpose: This paper takes a famous managerial model of decision-making in a non-medical setting and integrates it with a well- known model of medical decision-making to generate a unified illustration of the process. Both models deal with decision-making. However, Simon's model is less easily applied to the unique process of medical decision making. The proposed integration may help bridge the gap between the models and approaches by creating a unified framework to deal with the challenge of medical decision making in hospital environments through empirical methods.

Approach: Simon's model of automation provides the general structure of the decision-making process by dividing it into three stages: Intelligence, Design and Choice. The Pauker \& Kassirer model deals with probabilistic and statistical applications of clinical processes, and introduces a threshold approach and decision trees as the main decision tools. The discussion explores the advantages and disadvantages of each model and what can be gained by combining them.

Research limitations: Although these models were used to form an integrated framework, they were developed almost three decades apart. Therefore, caution is of the essence when applying them to real-life circumstances, and further research is needed to validate this integration.

\section{Key words}

Medical decision modeling, Medical decision making, Decision analysis, Probabilistic modeling

\section{I ntroduction}

\section{Overview of the problem}

The process of medical decision-making has a number of unique characteristics, which make it worth studying and modeling. It is often carried out in a challenging setting of tight time constraints and high levels of pressure and cognitive 
load, especially in emergency departments in hospitals ${ }^{[1]}$. Furthermore, there are great risks involved, so that maximizing decision efficiency and accuracy is vital. This is exacerbated by the fact that modeling medical decision making is immensely complex, as it takes multiple variables and potential influencers into account. Medical decision-making usually takes place in the context of a healthcare institution or organization. There are several theoretical and mathematical models that can be applied to the process of clinical decision-making. These models analyze alternatives and infer the probabilistic likelihood and risks faced by the decision-maker. Szolovits and Pauker ${ }^{[2]}$ developed an expert model of categorical and probabilistic reasoning. There have been several more recent attempts to develop knowledge-based diagnostic assistive tools ${ }^{[3-6]}$.

Pauker and Kassirer ${ }^{[7]}$ followed by Pauker and Kassirer ${ }^{[8]}$ as well as Simon ${ }^{[9]}$ put forward relatively comprehensive yet parsimonious models. Pauker and Kassirer's model narrowed down the decision-maker's alternatives in terms of testing and treating when facing a diagnostic dilemma, while conceptualizing the risks and benefits in statistical, computational terms. Simon's model broke down a managerial decision into three structural stages: Intelligence, Design and Choice.

The objective of this paper is to present these two models and then integrate them to provide a unified illustration of the clinical decision-making process. The combination of these models can assist medical decision-making processes by providing decision-makers with an empirical, theoretically-driven conceptualization of the key components of these processes and their interrelations.

We first introduce Simon's ${ }^{[9]}$ model, and present a general portrayal of its principles and fundamentals. We then discuss its applications to the field of medical decision-making and compare it to Pauker \& Kassirer's ${ }^{[7,8]}$ models before presenting the combined model of a decision-making process that relies on empirical, probabilistic tools. The complexities of implementing the model using decision trees and sensitivity analysis are discussed, followed by a general summary and conclusions.

\section{Simon's model (IDC model)}

Simon's ${ }^{[9]}$ model was designed to characterize the impact of automation technology on facets of manpower and management. The model was developed in the context of growing concerns regarding the replacement of human workers by automatons which could make human labor redundant. Simon refuted these claims by showing how similar trends, for instance the replacement of horse-drawn machines by mechanical equipment, was actually a rational decision rather than a forced change in reality. However, Simon believed that the introduction of new technological modes of production could have a considerable, less predictable impact on labor since automation was likely to alter the stages of managerial decision making. According to Simon's ${ }^{[9]}$ model, the manager - as a decision-maker - faces a choice in all aspects of managerial work. The model treats decision-making as synonymous to managing, but refers to it not merely as a choice among alternatives, but as a broader three-staged process. The stages form the acronym IDC - Intelligence, Design, Choice. The Intelligence stage is the initial locating of decision opportunities in which an alternative has to be chosen. The second stage - the design stage - is comprised of formulating possible courses of action for handling situations targeted during the intelligence stage. The third stage, in which one of the paths generated during the design stage is selected, is called the choice stage. Since its publication, Simon's ${ }^{[9]}$ model has been applied in numerous organizational and personal decision-making and policy-making studies ${ }^{[10,11]}$.

There are several limitations when trying to apply this model in healthcare settings. First of all, it was primarily formulated to describe decision-making processes at the organizational level and not as a single physician-patient clinical decision-making process. Second, it cannot describe a shared decision-making process (between the patient and the physician), despite the fact that today the Health 2.0 revolution is increasingly characterized by shared medical decision-making processes. 


\section{Pauker \& Kassirer's models for medical decision analysis}

Pauker \& Kassirer ${ }^{[7]}$ presented a model of medical decision-making that uses decision trees and sensitivity analyses to generate a more informed and accurate decision-making process. The model views medical decision-making as a process that involves a considerable amount of uncertainty and complexity, and thus employs a series of probabilities and thresholds to make it a more organized and fixed procedure.

This model is an application of an earlier one ${ }^{[12]}$ in which the authors developed a formula they called the therapeutic threshold which indicated the probability that disease would constitute the point of indifference between two clinical strategies. In problems that can be reduced to two choices, for instance either administering medical care or withholding a specific treatment for a specific disease, this threshold can be used as a solution. The optimal choices are to withhold treatment when the estimated probability of disease is below the therapeutic threshold, or to administer treatment when the probability is above this threshold. The therapeutic threshold is calculated from data describing the benefits and risks of the specific treatment. In their later model ${ }^{[7]}$ the authors extended the threshold concept to problems that can be reduced to three choices: withhold treatment, treat without testing, or perform a test that determines the subsequent approach using two threshold probabilities ("test-threshold" and "test-treatment threshold").

The model itself is presented in further detail here in section 4 as it relates to the implementation of the IDC model in healthcare.

There might be several limitations when attempting to apply this model in healthcare settings. First, no economic considerations are built into this model such as the utility value of each strategies, and no health-economic issues are raised or discussed. Second, the model does not take the costs of medical tests and the medical treatments into account. Similarly, it does not deal with the availability of resources/equipment needed to conduct the medical tests or treatments. Thus, the model, although contributing much to clinical decision-making, does not provide an optimal solution to the medical dilemma as a whole.

Hence, given the advantages and scope each of these models as regards the healthcare setting, combining them could lead to a more comprehensive, easily implementable model of decision making.

\section{I mplementing Simon's model (I DC model) and the Pauker-Kassirer model in the healthcare domain}

This section presents the implementation of Simon's Model (IDC Model) in the healthcare domain. The general flow chart of the model can be seen in this graph. The three stages of the IDC models are illustrated in this graph along with their major components (see Figure 1).

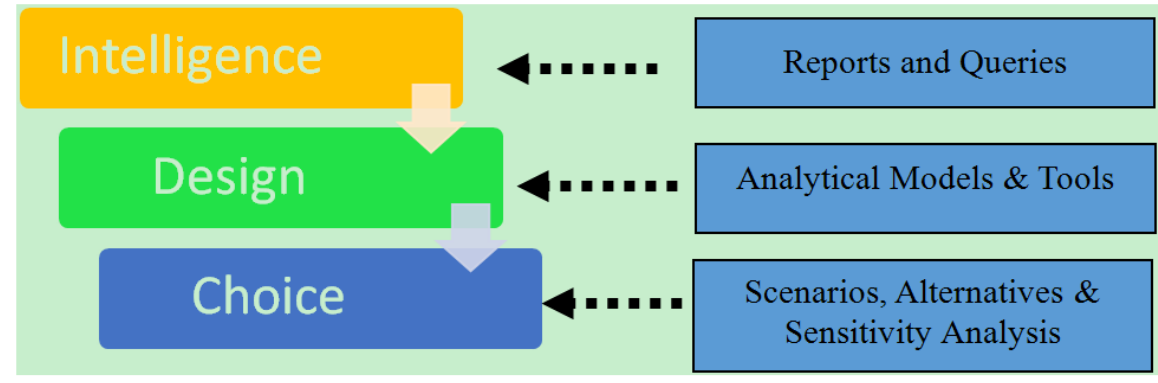

Figure 1. A general flowchart of Simon's model (adapted from Simon ${ }^{[9]}$ ) 


\subsection{The intelligence stage}

Of the three stages, the intelligence stage is the most elaborate and time-consuming. In the medical domain, this stage includes obtaining data regarding the patient's current complaint, the history of the problem, past medical history and the current state of the problem (e.g. previous hospitalizations, surgeries, permanent medications, lab results, drug sensitivity, etc.). For a physician examining the patient at a hospital, there are several possible sources of information pertaining to this stage. The immediate source is - naturally - the patient's complaints. Other sources include the collection and interpretation of basic physical measures (blood pressure, heart rate, blood sugar levels, etc.), data from electronic medical information systems in the institute or the referring organization ${ }^{[13,14]}$, history taking interviews conducted by medical staff members ${ }^{[15]}$ and updates from other physicians or institutes that have dealt with the patient. While this stage does not involve the physician's judgment regarding preferable choices or courses of action, it is the foundation for the stages to come. Information gathered during the intelligence stage is still in a descriptive, factual form and thus focuses on symptoms and complaints and not on defining the problem or embarking on complex diagnostic procedures.

\subsection{The design stage}

The design stage involves searching and evaluating treatment and testing options. At this stage the physician has three possible alternatives available: testing to gather more information, treating the patient without testing, and finally providing no treatment. Pauker \& Kassirer ${ }^{[8]}$ addressed this sort of choice in their model from a probabilistic point of view. Each alternative has certain probabilities of risks and/or advantages. For instance, subjecting a patient to an imaging test provides more information and allows for more informed decision-making, but imaging techniques may expose the patient to unhealthy levels of radiation. According to information gathered during the intelligence stage, the physician needs to compare the probability of a certain diagnosis to the different thresholds: If the probability of the diagnosis is below the testing threshold, the option of no treatment should be chosen. If the probability of the diagnosis is above the test-treatment threshold, suitable treatment for that diagnosis should be administered.

Finally, if the probability falls between the testing threshold and the test-treatment threshold, more tests should be performed, and the process should be repeated once test results are obtained. Applying Simon's ${ }^{[9]}$ model to the clinical decision-making process enables a rational and quantifiable strategy of choice in performing medical tests. The thresholds used in this process might be calculated as follows:

Testing threshold, as delineated in the original model ${ }^{[7]}$ :

$$
T_{t}=\frac{\left(P_{p o s / n d} \times R_{r x}\right)+R_{t}}{\left(P_{p o s / n d} \times R_{r x}\right)+\left(P_{p o s / d} \times B_{r x}\right)}
$$

Formula 1

Test-Treatment threshold ${ }^{[7]}$ :

$$
T_{\text {trx }}=\frac{\left(P_{\text {neg } / n d} \times R_{r x}\right)-R_{t}}{\left(P_{\text {neg } / n d} \times R_{r x}\right)+\left(P_{\text {neg } / d} \times B_{r x}\right)}
$$

Terms ${ }^{[7]}$ :

$P_{\text {posind }}$ - The probability of an incorrect positive test result (type one error).

$P_{p o s / d}$ - The probability of a correct positive test result.

$P_{n e g / n d}$ - The probability of a correct negative test result.

$P_{n e g / d}$ - The probability of an incorrect negative test result (type two error).

$B_{r x}$ - The benefit of treatment to a patient with the diagnosis.

$R_{r x}$ - The risk of treating a patient without the diagnosis.

$R_{t}$ - The risk of performing the test. 
Each alternative is then evaluated according to its applicability to a specific patient, and the decision-maker also considers the predicted outcomes of each alternative. Once the thresholds are calculated, the data gathered in the intelligence stage are processed into a decision tree with three types of nodes describing choices, chances and outcomes. In addition to enabling the calculation of the likelihood of each outcome, using the tree also yields a numerical expression of each outcome's relative worth on a specific scale. The expectancy of the general worth is calculated as a weighed mean of all possible outcomes, where the weight is the probability of each outcome. For example, a decision-maker faces two alternatives: conservative versus surgical treatment. The first alternative leads to a $10 \%$ probability of death (worth 0 ), a $30 \%$ probability of survival with a disabling condition (worth 0.7 ) and a $75 \%$ probability of survival in good health (worth 1). The second alternative leads to a $15 \%$ probability of death (worth 0 ), a $10 \%$ probability of survival with a disabling condition (worth 0.7 ) and an $85 \%$ probability of survival in good health (worth 1 ).

The general expected utility of the first alternative is 0.96 , while that of the second alternative is 0.92 . Thus, according to Expected Utility theory (which assumes that the decision-maker makes rational decisions), the first alternative would represent a preferable choice. In the case of a different decision maker who views a disabling condition as worth 0.4 , for instance, the second alternative would be better.

The main advantage of this method is that it enables the decision-maker to alter the data of part of the tree's (sub-trees) worth and probability depending on the diagnosis and the specific characteristics of the patient. While diagnoses and tests change across cases, the structure of the decision tree remains more constant. For instance, as Pauker \& Kassirer ${ }^{[8]}$ showed, the likelihood of discovering a cancerous diagnosis in an X-ray of a benign lesion is lower than the likelihood of discovery in a palpable lesion. In addition, the likelihood of reoccurrence of cancer when the patient complains of a small lump is ten times higher than the likelihood of cancer being identified in a general X-ray. These are also examples of how the structure of the decision process remains the same whereas the probabilities and worth of the outcomes change.

One of the method's most significant advantages is that by viewing a decision tree, physicians can make decisions in a more empirical way, even when under severe time constraints (e.g. when a certain treatment requires a relatively slow testing procedure, and the physician is unsure of how much time the patient can remain stable without deteriorating, a decision tree may assist the physician in generating a clinical strategy while waiting for the test results). It is important to keep in mind that this method should not be applied strictly, and should be used as an assistive tool. Strict application of Expected Utility theory may lead to results that may seem implausible, thus resulting in further demands on physician resources ${ }^{[16]}$.

\subsection{The choice stage}

The choice stage introduces the chosen alternative, and the application of sensitivity analyses. Real-world events rarely involve such narrowly-focused changes, since they do not give a complete picture and leave the decision-maker at a considerable level of uncertainty ${ }^{[17]}$. This is why a sensitivity analysis on the results may make sense ${ }^{[18]}$. In a sensitivity analysis, the decision-maker changes one (or more) of the values assigned to the components of the decision tree to determine whether the optimal decision will change. A sensitivity analysis, also known as a "what if" analysis, formulates questions such as "What if the probability of the disease is greater?", "What if the test is less likely to be accurate?", etc. In its simplest form a sensitivity analysis changes only one variable, while other variables remain constant.

A one-way sensitivity analysis is illustrated below on the decision to perform an MRI test for suspected cancer or to administer chemotherapy first (before or without testing the patient). It is presented in the following graph (see Figure 2).

The intersection point between these two strategies can provide information on the preferred choice. For instance, if the likelihood of discovering a cancer in a specific MRI test is above the intersection point (above 22.5 percent), the MRI test should be performed before providing any treatment, or administer chemotherapy first. It is understood that there could be more clinical strategies, which cannot necessarily be modeled as linear. 


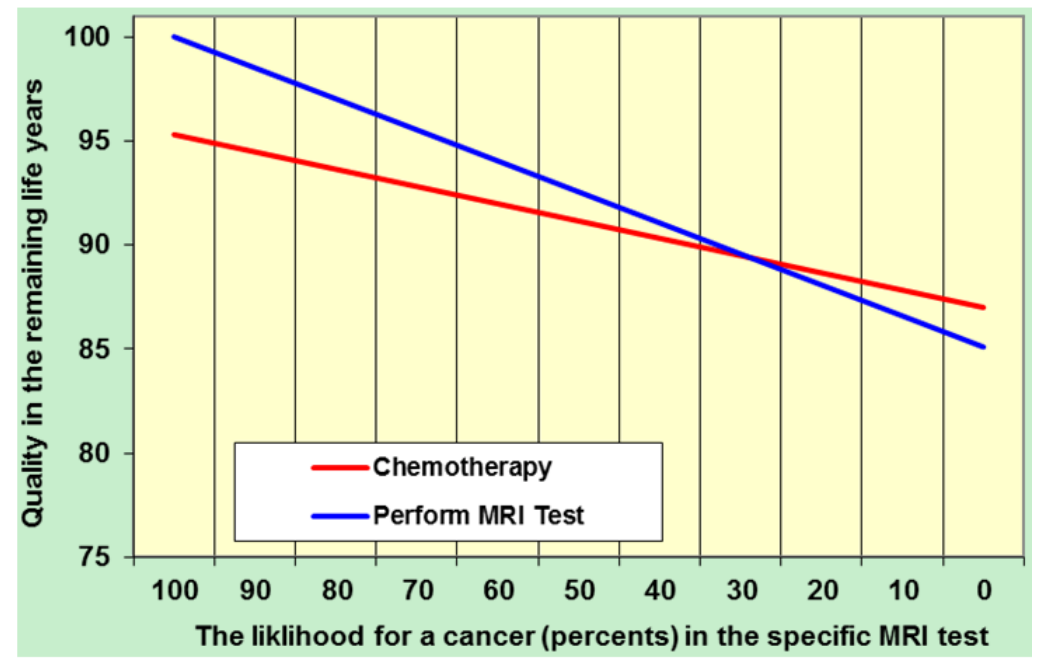

Figure 2. Illustration of a one-way sensitivity analysis

In most cases the decision-maker will choose to conduct a more complex sensitivity analysis that models the consequences of several changes and can create a more valid emulation of medical reality ${ }^{[19-21]}$. Because sensitivity analyses that strive to thoroughly model real process are extremely time-consuming, several technological aids have been developed to automate this procedure. These include applications of electronic worksheets (such as Microsoft Excel) or shelf products designed specifically for this purpose (e.g. "Expert Choice- Decision Trees" and "Treeage pro").

The choice stage may strike the reader as the simplest of them all, but Neyman and Zviran ${ }^{[21]}$ showed why for several reasons, it is quite complex:

Multiple Preferences - This concerns a potential contradiction between two alternatives; i.e., the proposed treatment versus the patient's preference. This issue cannot be dealt with mathematically and requires efforts outside the framework of the model, such as dialoguing with the patient to reach an agreement regarding the available options, or presenting the patient with alternatives along with the risks and benefits (i.e. a life expectancy of eight years with a normal voice versus a life expectancy of ten years with speech impairments). In addition, the patient can be presented - through a Markovian process - a combined model of possibilities and the probabilities of their occurrence in terms of years of life, thus providing the patient with the number of quality-adjusted life years (QALY) for each alternative ${ }^{[22]}$.

Uncertainty - Medical problems may go beyond the determinism that characterizes probabilistic modeling, and the physician often has to use subjective judgment of risk-probability, thus increasing the uncertainty of the final decision.

Conflicting interests - This point does not concern the issue of thresholds directly. Rather, it is a common situation where the decision made by the physician may be subject to conflicting interests that can be financial, medical or related to the hospital's policies.

Control - Before making the final decision, there must be verification that it is controllable, and that all possible alternatives have been weighed and thought of, should the chosen alternative fail.

Multiple decision makers - The entire process becomes even more complex when several opinions and perspectives are taken into account, in particular, when a group of physicians try to reach a joint decision. While patients are often advised to consult several physicians from different fields of expertise for a second opinion, it may also be wise to entrust the final decision to the main care- giving physician, who will weight all the different alternatives. 


\section{I mplementing Simon's model using decision trees}

The use of decision trees has some inherent flaws, one of which has to do with assigning worth values to different medical outcomes. This is problematic due to the lack of an objective evaluation of the outcome. Assigning worth to an outcome has mathematical benefits when modeling a decision-making process, but it rarely translates into real outcomes in terms of worth and advantages for the patient. The literature provides several explanations for this failure.

Life expectancy - The vast majority of cases in the literature depict the average patient, and not the specific patient the physician is dealing with when turning to a decision tree model. Therefore, the physician must add her/his own subjective estimation of the patient's chances of survival and life expectancy, which, in fact, turns a supposedly rational and empirical process into one that is subject to personal interpretation and beliefs. According to Beck et al. ${ }^{[23]}$ the approximation of life expectancy is a negative exponential function which in itself has several prediction structures. This makes the picture even more complex for a physician attempting to apply a decision tree model.

Quality of Life - The decision maker is indeed the physician, but she/he must collaborate with the patient in this process. The physician's knowledge and clinical experience must meet the patient's expectations and adjust for the implications of the medical outcome on the individual and the family. Therefore, this must be made into an easy-to-understand process for the patient, and her/his views must be taken into account, thus compromising the "empirical" nature of the process. One way to make the process accessible to the patient is to present her/his with the possible scenarios and ask her to rank them according to her preferences. Another way is to present her with the predicted length of the scenario as a way to demonstrate this trade-off ${ }^{[24]}$.

The QALY Measure - QALY is a standard measure of quality-of-life and life expectancy. It is a weighted average of both quality and quantity, and is used to measure the benefits and effectiveness of improvements and developments in the medical field ${ }^{[25]}$. QALY can be used to guide decision makers on both the patient level and the ward/hospital policy level. The developer of this method, Allen Williams, characterized it as taking both factors (quality of life and predicted life expectancy) into account, while reflecting the values and moral standards of the society which it serves ${ }^{[26]}$. There is no consensus regarding the maximal cost of one additional QALY unit, which makes a decision worth adopting. The cost-effectiveness ratio does allow for a better review and consideration of the course of action in question. For instance, in the US it is common among policy-makers to view solutions whose cost is less than $\$ 50,000$ per QALY unit as feasible ${ }^{[27]}$.

Normative Cost-Effectiveness analysis - QALY may be used as a measure in the assessment of the normative value of information in a hospital whose policy makers are debating the adoption of a new medical treatment or medical information system. The feasibility of the system is determined by the cost associated with one QALY unit contributed by the system. Mathematically, this would be $\Delta$ Costs/AQALYs. Practically, such an assessment would involve constructing decision-trees that reflect the typical routine activities and decision junctions in the specific point of care with the worth of each outcome. While taking into account the cost of implementing the system and the number of patients treated in that point of care, the feasibility of a solution can be evaluated.

\section{Summary and conclusions}

This presentation of a common framework to discuss Simon's ${ }^{[9]}$ and Pauker \& Kassirer's ${ }^{[7,8]}$ models sought to highlight some important approaches in empirical medical decision-making, and illustrate their shortcomings while offering potential strategies to cope with them. Each model has its shortcomings when used alone: Simon's model provides a constructed conceptualization of management-level decision-making in organizations unrelated to the healthcare sector, but it is less applicable to the individual user (such as a single clinician or any clinical staff), whereas Pauker \& Kassirer's model may be applied to physicians, but its relevance to policy makers and managerial decision-makers in the healthcare 
sector is less clear although this model can be more structural and divided into stages similar to Simon's model. Combining these two models serves to create a common, unified framework for decision-making processes in healthcare settings.

The hospital sector today is far from able to implement a fully reliable tool that can provide a definitive conclusion as to a preferred course of action a clinician should take. However, the use of methods such as the ones outlined here, alongside actual changes in the healthcare system that would enable their use, may make the process more efficient, and allow clinicians to spend less time and resources on decision making, without undermining their effectiveness. Introducing a combined model that deals with actual procedures (testing, imaging, treatment) and uses a computational and statistical perspective weighted against potential outcomes and their probabilities, can enable clinicians and/or managerial policy-makers to take into account the worth of each alternative when discussing optional courses of action (i.e. the accuracy of testing versus the potential effectiveness in terms of QALY).

The process of medical decision-making poses some substantial challenges to the science of decision-making. Clinical training does not necessarily make this process less of an unmarked territory and in hospitals it is often carried out in less than optimal conditions. Yet, it still calls for efficient, empirical methods to assist clinicians in making informed, statistically valid decisions.

Although these models were used to form an integrated framework, they were developed by very famous researchers from different fields of expertise, and almost three decades apart. Therefore, caution is of the essence when applying them to real-life circumstances, and further research is needed to validate this integration. Future work with the proposed model should test its applicability. This testing should simulate real-time decision making first, and then move on to actual decision-making. The issues raised by Neyman \& Zviran ${ }^{[21]}$ should be taken into account when testing the model, as should the issues regarding the use of QALY and related measures. The latter point is of great significance, since one of the major directions needed to further develop this model for new research or even develop it into a fully functional decision support tool has to do with the integration of the costs of using various strategies, including their medical tests and treatments so that their advantages and disadvantages can be better assessed, and the decision made more cost effective.

\section{Competing interests}

The author declared that he had no significant competing financial, professional, or personal interests that might have influenced the performance or presentation of the work described in this manuscript.

\section{References}

[1] Laxmisan, A, Hakimzada, F, Sayan, OR, Green, RA, Zhang, J, Patel VL. The multitasking clinician: decision-making \& cognitive dem\& during \& after team h\&offs in emergency care, International journal of medical informatics. 2011; 76(11): 801-811.

[2] Szolovits, P, Pauker SG. Categorical and probabilistic reasoning in medical diagnosis. Artificial Intelligence. 1978; 11(1): 115-144. http://dx.doi.org/10.1016/0004-3702(78)90014-0

[3] Shortliffe EH. Medical expert systems-knowledge tools for physicians. Western Journal of Medicine. $1986 ; 145(6)$ : 830-839. PMid: 3811349.

[4] Miller RA. Medical Diagnostic Decision Support Systems-Past, Present, And Future A Threaded Bibliography and Brief Commentary. Journal of the American Medical Informatics Association. 1994; 1(1): 8-27. PMid: 7719792. http://dx.doi.org/10.1136/jamia.1994.95236141

[5] Croskerry P. A universal model of diagnostic reasoning. Academic Medicine. 2009; 84(8): 1022-1028. PMid: 19638766. http://dx.doi.org/10.1097/ACM.0b013e3181ace703

[6] Ocak H. A Medical Decision Support System Based on Support Vector Machines and the Genetic Algorithm for the Evaluation of Fetal Well-Being.Journal of medical systems. 2013; 37(2): 1-9. PMid: 23321973. http://dx.doi.org/10.1007/s 10916-012-9913-4

[7] Pauker, SG, Kassirer JP. Medical progress: Decision analysis, New Engl \& Journal of Medicine. 1980; 316(5): 250-258. PMid: 3540670. http://dx.doi.org/10.1056/NEJM198701293160505 
[8] Pauker, SG, Kassirer, JP. The threshold approach to clinical decision making, The New Engl \& Journal of Medicine. 1987; 302(20): 1109-1117. PMid: 7366635. http://dx.doi.org/10.1056/NEJM198005153022003

[9] Simon, HA. The Shape of Automation for Men \& Management. New York: Harper \& Row. 1965.

[10] Simon, HA. Problem forming, problem finding \& problem solving in design, Design \& systems. 1995; 245-257.

[11] Salles, M. Decision making in SMEs \& information requirements for competitive intelligence, Production Planning \& Control. 2006; 17(3): 229-237. http://dx.doi.org/10.1080/09537280500285367

[12] Pauker SG, Kassirer JP. Therapeutic decision making: a cost-benefit analysis, New Engl \& Journal of Medicine. 1975; 293: 229-34. PMid: 1143303. http://dx.doi.org/10.1056/NEJM197507312930505

[13] Ben-Assuli, O, Shabtai, I, Leshno, M. The impact of EHR \& HIE on reducing avoidable admissions: controlling main differential diagnoses, BMC Medical Informatics \& Decision Making. 2013; 13(1): 49-59. PMid: 23594488. http://dx.doi.org/10.1186/1472-6947-13-49

[14] Ben-Assuli, O., Leshno, M. Using Electronic Medical Records in Admission Decisions: A Cost Effectiveness Analysis, Decision Sciences. 2013; 44(3): 463-481. http://dx.doi.org/10.1111/deci.12018

[15] Witting, MD, Hayes, BD, Schenkel, SM, Drucker, CB, DeWane, MP, Lantry III, JH, et al. Emergency Department Medication History Taking: Current Inefficiency \& Potential for a Self-Administered Form. The Journal of emergency medicine. 2013; (In press). http://dx.doi.org/10.1016/j.jemermed.2013.01.019

[16] Buchholz, W, Schymura, M. Expected utility theory \& the tyranny of catastrophic risks, Ecological Economics. 2013 ; 77: $234-239$. http://dx.doi.org/10.1016/j.ecolecon.2012.03.007

[17] Petrou, S, Gray, A. Economic evaluation using decision analytical modelling: design, conduct, analysis, \& reporting, British Medical Journal. 2011; 342(11): d1766.

[18] Baio, G, Dawid, AP. Probabilistic sensitivity analysis in health economics, Statistical methods in medical research. 2011; http://smm.sagepub.com/content/early/2011/09/17/0962280211419832. http://dx.doi.org/10.1177/0962280211419832

[19] Koerkamp, BG, Weinstein, MC, Stijnen, T, Heijenbrok-Kal, MH, Hunink MM. Uncertainty \& patient heterogeneity in medical decision models. Medical Decision Making. 2010; 30(2): 194-205. PMid: 20190188. http://dx.doi.org/10.1177/0272989X09342277

[20] Nutt, DJ, King, LA, Phillips, LD. Drug harms in the UK: a multicriteria decision analysis, The Lancet. 2010; 376(9752): 1558-1565. http://dx.doi.org/10.1016/S0140-6736(10)61462-6

[21] Neyman, S, Zviran M. Information Systems - From Theory to Practice. 2001; Tel Aviv: Dyonon, Tel-Aviv University.

[22] Beck, JR, Pauker, SG. The Markov process in medical prognosis, Medical Decision Making. 1983; 3(4): 419-458. PMid: 6668990. http://dx.doi.org/10.1177/0272989X8300300403

[23] Beck, JR, Kassirer, JP, Pauker, SG. A convenient approximation of life expectancy (the "DEALE"): I. Validation of the method, The American journal of medicine. 1982; 73(6): 883-888. http://dx.doi.org/10.1016/0002-9343(82)90786-0

[24] McNeil, BJ, Weichselbaum, R, Pauker, SG. Speech \& survival: tradeoffs between quality \& quantity of life in laryngeal cancer, The New Engl\& journal of medicine. 1981; 305(17): 982-987. PMid: 7278922.

http://dx.doi.org/10.1056/NEJM198110223051704

[25] Phillips, C, Thompson, G. What is a QALY? 1998; 1(6). Hayward Medical Communications.

[26] Williams, A. QALYs \& ethics: a health economist's perspective. Social Science \& Medicine. 1996; 43(12): $1795-1804$. http://dx.doi.org/10.1016/S0277-9536(96)00082-2

[27] Grosse, S D. Assessing cost-effectiveness in healthcare: history of the \$50,000 per QALY threshold. Exp Rev of Pharmacoeconomics \& Outcomes Res. 2008; 8(2): 165-178. PMid: 20528406. http://dx.doi.org/10.1586/14737167.8.2.165 